\title{
Antifibrotic potential of bone marrow-derived mesenchymal stem cells in biliary atresia mice
}

\author{
JUN LEI ${ }^{1}$, YONG CHAI $^{1}$, JUHUA XIAO ${ }^{2}$, HUAKUN HU $^{1}$, ZHIQIANG LIU $^{1}$, YU XIAO $^{1}$, \\ LIJUN YI $^{1}$, JINSHI HUANG ${ }^{1}$, TIANXIN XIANG ${ }^{3}$ and SHOUHUA ZHANG ${ }^{1}$ \\ ${ }^{1}$ Department of General Surgery, Jiangxi Provincial Children's Hospital; ${ }^{2}$ Department of Ultrasound, \\ Jiangxi Provincial Maternal and Child Health Hospital; ${ }^{3}$ Department of Infectious Disease, \\ The First Affiliated Hospital of Nanchang University, Nanchang, Jiangxi 330006, P.R. China
}

Received February 7, 2018; Accepted July 27, 2018

DOI: $10.3892 / \mathrm{mmr} .2018 .9353$

\begin{abstract}
Biliary atresia (BA) is a rare and severe disease that affects infants where a fibroinflammatory process destroys the bile ducts, leading to fibrosis and biliary cirrhosis, and mortality if untreated. Bone marrow-derived mesenchymal stem cells (BMMSCs) have been considered as a promising therapy in fibrotic diseases. The aim of the present was to investigate the anti-fibrotic roles of BMMSC transplantation in a BA mouse model. Mouse BA models were established by Rhesus rotavirus administration to neonatal mice. The results revealed that the liver enzyme and bilirubin metabolism levels, and the levels of the oxidative stress marker malondialdehyde (MDA) and the fibrosis marker were all increased in the BA model, while the liver tissue levels of superoxide dismutase and glutathione peroxidase were reduced. The hematoxylin and eosin and Masson's trichrome staining revealed severe liver fibrosis and collagen accumulation in BA livers. However, these indicators were all reversed once the BA mice were administered the BMMSC inoculation. In conclusion, the present study demonstrated the anti-fibrotic potential of BMMSCs in BA mice, which may provide a novel approach to ameliorate the fibrotic response in BA patients.
\end{abstract}

\section{Introduction}

Biliary atresia (BA), a unique disease of infancy, is caused by fibro-inflammatory destruction of extra- or intra-hepatic bile

Correspondence to: Dr Shouhua Zhang, Department of General Surgery, Jiangxi Provincial Children's Hospital, 122 Yangming Road, Nanchang, Jiangxi 330006, P.R. China

E-mail: zshouhua416@163.com

Dr Tianxin Xiang, Department of Infectious Disease, The First Affiliated Hospital of Nanchang University, 17 Yongwai Road, Nanchang, Jiangxi 330006, P.R. China

E-mail: txxiangmed@163.com

Key words: bone marrow-derived mesenchymal stem cells, biliary atresia, liver fibrosis, oxidative stress, rhesus rotavirus ducts. BA leads to cholestasis and progressive liver fibrosis and cirrhosis, as well as end-stage liver disease in some infants, who then require a liver transplantation $(1,2)$. Worldwide, the incidence of BA ranges from 1 in 5,000 to 1 in 20,000 live births, which is responsible for nearly one-third of all neonatal cholestasis cases and more than $90 \%$ of obstructive cholestatic cases (3). If untreated, the prognosis is extremely poor, with death from liver cirrhosis occurring within 2 years (4). Although a type of surgery knows as the Kasai procedure has markedly improved the prognosis of BA, life quality of the majority of patients remains relatively poor, and long-term survival depends on a liver transplant (5).

Over the past decade, mesenchymal stem cells (MSCs) have been extensively investigated as potential therapeutic options for the treatment of various degenerative diseases and immune disorders $(6,7)$. Focus on MSCs lies in their potential for self-renewal, ability to differentiate into multiple cell lineages, and immunoregulatory properties, as well as their ability to secrete several types of anti-fibrotic molecules (8), such as hepatocyte growth factor (9). Furthermore, unlike embryonic stem cells, the use of MSCs does not give rise to ethical issues, and they have a safer profile in terms of carcinogenesis. To date, the effects of bone marrow-derived MSCs (BMMSCs) on liver fibrosis induced by BA remain largely unknown. In the present study, we investigated the anti-fibrotic potential of BMMSCs in a murine model of BA induced by RRV administration.

\section{Materials and methods}

Ethics statement. The present study was reviewed and approved by the Ethics Committee of the Jiangxi Provincial Children's Hospital (Jiangxi, China) and all of the experiments were carried out in accordance with relevant guidelines and regulations. All procedures adhered to the Chinese Veterinary Medical Association Guide.

Animals. Pregnant BALB/c mice aged 10 to 12 weeks and 4-week-old healthy BALB/c nonpregnant mice were purchased from SLAC Inc. (Shanghai, China). The animals were housed in groups in a specific pathogen-free facility under artificial lighting conditions (12:12 h), with food and water available ad libitum. 
Murine BA model induced by RRV. A RRV strain (MMU 18006) was purchased from the American Type Culture Collection (Manassas, VA, USA), and amplified in MA104 cells. The experimental model of BA was established as described in previous study (10). Briefly, neonatal mice were injected with $20 \mu 1$ of $1.2 \times 10^{5} \mathrm{pfu} / \mathrm{ml}$ of RRV or supernatant of MA104 cell culture medium (controls) intraperitoneally within $24 \mathrm{~h}$ of birth. Infected mice that died within the first 2 days or that were not fed by their mothers were excluded from further analysis. All mice were weighed every day and observed the signs of cholestasis (icterus of non-fur-covered skin, color and quality of stools, and bilirubin in urine) until the day of sacrifice. Liver samples were collected for histological analysis and western blot analysis.

BMMSC isolation, purification, and characterization. BMMSCs were obtained from 4-week-old BALB/c mice. BMMSC isolation and purification protocols were performed in accordance with method described previously (11). To exclude hematopoietic stem cells and leucocytes, a magnetic bead-based mouse cell depletion kit (Miltenyi Biotec, Bergisch Gladbach, Germany) containing anti-CD 45 , anti-CD11b, anti-CD5, anti-Gr1 (Ly-6/C), and anti-TER 119 monoclonal antibodies was used. The expression profiles of BMMSC surface markers were determined by flow cytometry. BMMSC plasticity (i.e., differentiation into chondrogenic, adipogenic, and osteogenic lineages) was assessed using a commercial kit (StemPro; Thermo Fisher Scientific, Inc., Waltham, MA, USA), following the manufacturer's instructions. The purified cells were kept in standard culture media until the day of transplant.

BMMSC transplantation. Infected mice received an intraperitoneal injection of $1 \times 10^{6}$ BMMSCs in a single dose 7 days post-challenge. All the animals included in the experimental groups were sacrificed via broking the neck 14 days post-inoculation, and livers were harvested for further studies.

Collection of blood and tissue samples. After 14 days of the inoculation, mice were intraperitoneally injected $1 \%$ pentobarbital sodium at $80 \mathrm{mg} / \mathrm{kg}$, then blood samples were withdrawn from the ventral main vein of the mice and allowed to clot. Serum was separated by centrifugation at 2,000 rpm for $10 \mathrm{~min}$ at $4^{\circ} \mathrm{C}$ and used for the assessment of liver function. Afterwards, the animals were killed by cervical dislocation. The livers were dissected out and portions placed in $10 \%$ neutral buffered formalin ( $\mathrm{pH}$ 7.4) for subsequent histopathological examination. The remaining tissues were kept deep frozen at $-80^{\circ} \mathrm{C}$.

Measurements of clinical biochemistry parameters. Murine blood samples were collected after the experiments and analyzed by the hospital's clinical laboratory using a Hitachi Pre-Analytical Process Automation System with a 7600 Clinical Analyzer (Hitachi, Ltd., Tokyo, Japan). The parameters analyzed were alanine aminotransferase (ALT), aspartate aminotransferase (AST), total bilirubin (TBIL), and direct bilirubin (DBIL).

Western blot analysis. Frozen liver samples were lysed with RIPA III lysis buffer (Sangon Biotech, Shanghai, China). After determination of the protein concentration with a BCA kit (Thermo Fisher Scientific, Inc.), equal amount of proteins from different samples were subjected to 10\% SDS-PAGE gels following electrophoretic transfer to nitrocellulose membranes. The membranes were then blocked with $5 \%$ nonfat milk and incubated with the primary antibodies against laminin $(\mathrm{LN})$, transforming growth factor- $\beta$ (TGF- $\beta$ ), tumor necrosis factor- $\alpha$ (TNF- $\alpha$ ), collagen IV (all Abcam, Cambridge, MA, USA, $\alpha$-smooth muscle actin ( $\alpha$-SMA; Cell Signaling Technology, Inc., Danvers, MA, USA), $\alpha-1$ type 1 collagen (COL1A1) (Abcam), or $\beta$-actin (Cell Signaling Technology, Inc.). After incubation with corresponding horseradish peroxidase-coupled secondary antibody (Beyotime Institute of Biotechnology, Haimen, China), the membrane was developed using an enhanced chemiluminescence system (Bio-Rad Laboratories, Inc., Hercules, CA, USA). Densitometric analysis was performed using ImageJ software (National Institutes of Health, Bethesda, MD, USA), using $\beta$-actin as an endogenous control.

Measurement of antioxidant enzyme activities. Liver tissues were homogenized in saline using a Teflon homogenizer (Glas Col, Vernon Hills, CA, USA). The sample was then centrifuged at 3,000 rpm for $15 \mathrm{~min}$ at $4^{\circ} \mathrm{C}$, and the resultant supernatant was collected. The homogenate was then used for assessments of oxidative stress markers. Antioxidant enzyme activities were measured using an SOD, GSH, and MDA activity detection kit (Nanjing Jiancheng Bioengineering Institute, Nanjing, China), following the manufacturer's instructions.

Histology and immunohistochemistry. Liver specimens were fixed in $10 \%$ neutral-buffered formalin and embedded in paraffin. Then, the embedded tissues were cut into $5 \mu \mathrm{m}$-thick serial sections. Next, the sections were deparaffinized in xylene, hydrated through graded ethanol, and stained with hematoxylin and eosin (H\&E) or Masson's trichrome for histopathological analysis. For immunohistochemistry, a three-step immunohistochemical protocol was used, including sectioning, dewaxing, and hydrating the tissues. After deparaffinized with xylene and rehydrated with graded ethanol, paraffin sections of tissues were antigen repaired with Tris-EDTA, followed by incubation with $3 \% \mathrm{H}_{2} \mathrm{O}_{2}$ at room temperature for $10 \mathrm{~min}$. Then, the slides were blocked with 5\% goat serum (diluted in PBS) for $1 \mathrm{~h}$ at room temperature and incubated overnight at $4^{\circ} \mathrm{C}$ with primary antibody against collagen IV, LN, $\alpha$-SMA, COL1A1, TGF- $\beta$ or TNF- $\alpha$ at the indicated dilutions, followed by incubation with secondary antibody and rinsing with PBS. Cell nuclei were counterstained with Harris hematoxylin solution.

Statistical analysis. Statistical analysis was performed using SPSS 23.0 software (IBM Corp., Armonk, NY, USA). Data were expressed as the mean \pm standard deviation. One-way analysis of variance followed by Tukey's post-hoc test were used to compare the means of multiple groups. $\mathrm{P}<0.05$ was considered to indicate a statistically significant difference.

\section{Results}

Liver enzymatic function and bilirubin metabolism recovered after BMMSC treatment. Liver enzyme levels such as AST, ALT, and the levels of bilirubin metabolism TBIL and DBIL were upregulated in the RRV-induced BA murine model as 

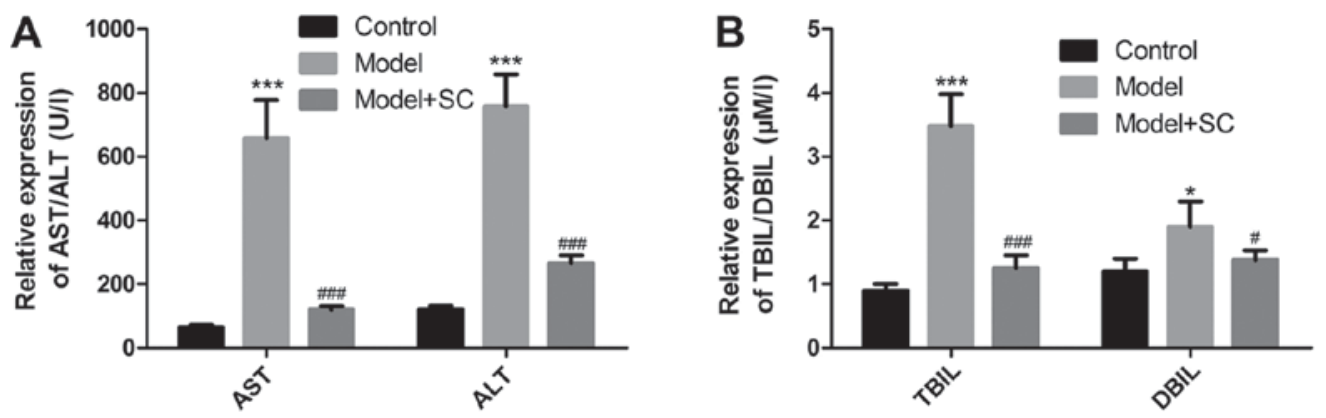

Figure 1. Evaluation of clinical biochemical parameters in blood samples from mice. Assessment of levels of (A) liver enzymes and (B) bilirubin metabolism $(\mathrm{n}=10) .{ }^{*} \mathrm{P}<0.05$ and ${ }^{* * *} \mathrm{P}<0.001$ vs. control group; ${ }^{\#} \mathrm{P}<0.05$ and ${ }^{\# \# \#} \mathrm{P}<0.001$ vs. BA model group. ALT, alanine aminotransferase; AST, aspartate aminotransferase; TBIL, total bilirubin; DBIL, direct bilirubin; SC, bone marrow-derived mesenchymal stem cells; BA, biliary atresia.
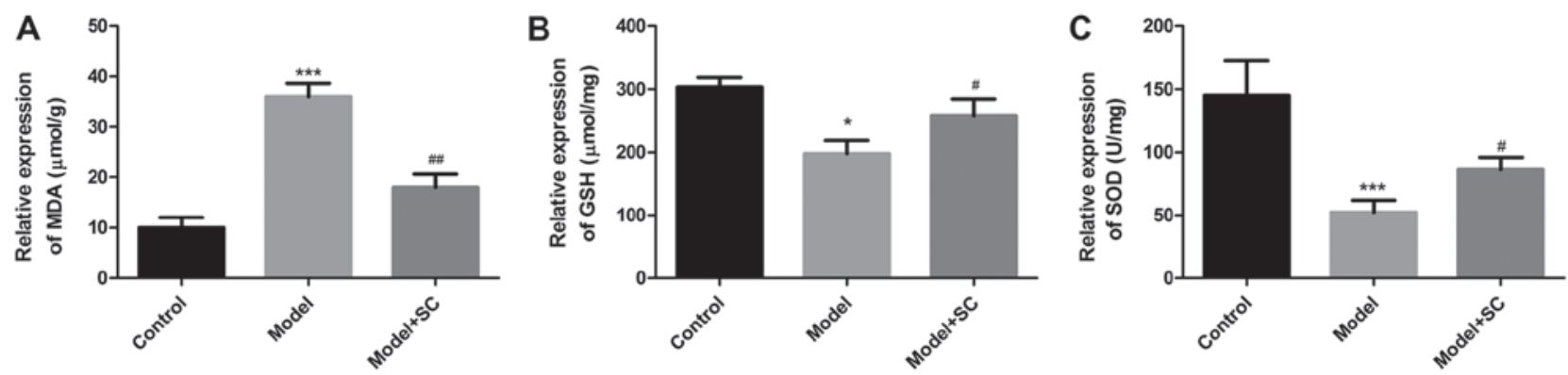

Figure 2. Detection of antioxidant enzyme activities in liver tissues of mice in Control, BA (model group), and BA + BMMSC (model + SC) groups. (A) MDA, (B) GSH and (C) SOD ( $\mathrm{n}=10)$. ${ }^{*} \mathrm{P}<0.05$ and ${ }^{* * * *} \mathrm{P}<0.001$ vs. control group; ${ }^{\#} \mathrm{P}<0.05$ and ${ }^{\# \#} \mathrm{P}<0.01$ vs. BA model group. MDA, malondialdehyde; SOD, superoxide dismutase; GSH, glutathione peroxidase; BMMSC/SC, bone marrow-derived mesenchymal stem cells; BA, biliary atresia.

compared with the control group (Fig. 1). Following BMMSC transplantation, liver enzyme and bilirubin metabolism levels markedly decreased in the RRV-induced BA murine model (Fig. 1), indicating that the BMMSCs greatly improved liver function.

Effect of BMMSC treatment on liver oxidative stress. As shown in Fig. 2, MDA levels significantly increased and GSH and SOD levels significantly decreased in the BA model as compared with those of the control group. BMMSC treatment reversed these effects, with clearly reduced MDA levels and increased GSH and SOD levels as compared with those in the nontreated RRV-induced BA group. These data indicated that BMMSC treatment dramatically inhibited liver oxidative stress in RRV-induced BA mice.

BMMSC treatment reduced the expression of proteins associated with liver fibrosis. Expression levels of collagen IV, LN, $\alpha$-SMA, COL1A1, TGF- $\beta 1$ and TNF- $\alpha$ in liver tissues increased significantly in the BA group when compared with those in the control group (Fig. 3). And BMMSCs treatment significantly decreased the protein expression of collagen IV, LN, $\alpha$-SMA, COL1A1, TGF- $\beta 1$, and TNF- $\alpha$ levels (Fig. 3 ). Moreover, results from immunohistochemical staining of collagen IV, LN, $\alpha$-SMA, COL1A1, TGF- $\beta 1$, and TNF- $\alpha$ proteins in liver tissues from the different groups (control group, BA group, and BA + BMMSC treatment group) were consistent with those assessed by western blotting (Fig. 4). Taken together, these results indicated that BMMSCs administration appeared to relieve liver fibrosis caused by BA.
The effect of the BMMSC treatment on histology. Liver sections of the control group displayed normal histological features, whereas liver sections in the BA group showed massive diffuse and progressive histological alterations, with massive mononuclear cellular infiltration and marked fibrosis (Fig. 5A and B). In the BMMSC-treated group, most of the histological alterations observed in the BA group disappeared, and cell histology was more or less similar to that observed in the control group (Fig. 5A and B). These findings suggested that BMMSC treatment alleviated liver fibrosis in a murine model of RRV-induced BA.

\section{Discussion}

BA is a devastating disease of newborns that occurs within the first few months after birth. The disease is characterized by liver inflammation, fibrosis, and obstruction of the bile duct (12). Although the etiology of BA is largely unclear, human and mouse data have implicated that a perinatal viral infection is a possible pathogenic mechanism (13). In the murine model of BA, RRV infection of newborn BALB/c mice results in inflammatory cholangiopathy, with a pathological phenotype similar to that seen in human BA $(14,15)$. BA mice develop bilirubinuria and jaundice, with pale-colored stools and growth retardation, culminating in death. Based on this, in the present study, we constructed a murine model of BA by RRV administration and explored the antifibrotic roles of BMMSCs in BA mice.

The present study demonstrated that BMMSCs treatment significantly restored liver enzymatic function and bilirubin 

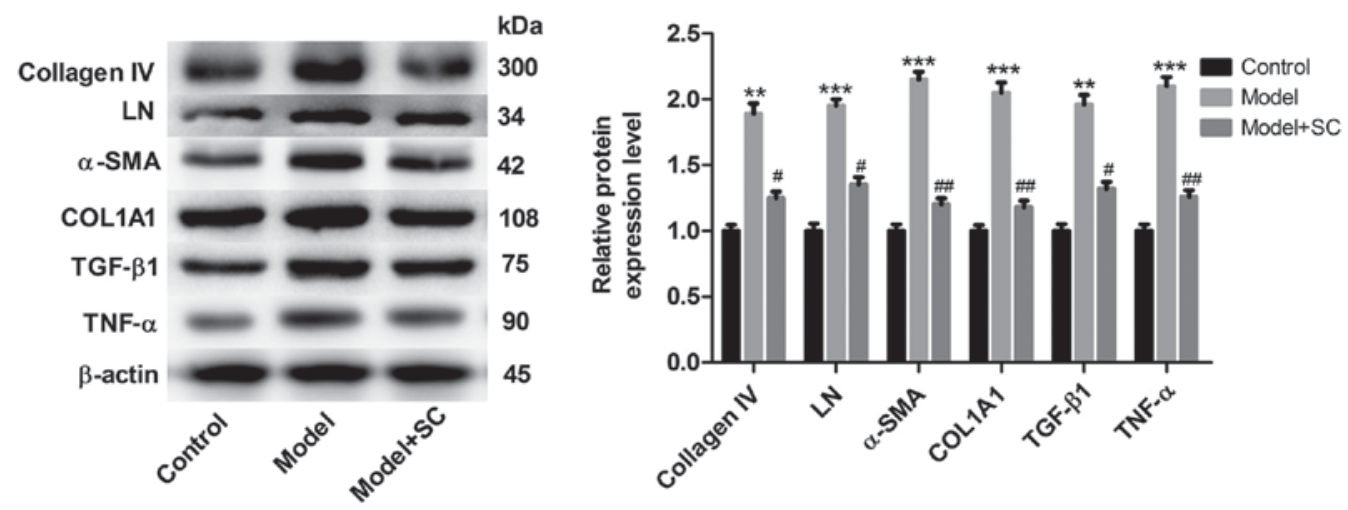

Figure 3. Detection of the expression of proteins associated with liver fibrosis by western blotting. Protein expression in liver tissues from the different groups: Control, BA model and BA + BMMSC (model + SC). $\beta$-catenin was used to normalize protein expression $(\mathrm{n}=10) .{ }^{* *} \mathrm{P}<0.01$ and ${ }^{* * *} \mathrm{P}<0.001 \mathrm{vs}$. control group; ${ }^{\#} \mathrm{P}<0.05$ and ${ }^{\# \#} \mathrm{P}<0.01$ vs. BA model group. $\mathrm{LN}$, laminin; TGF- $\beta$, transforming growth factor- $\beta$; TNF- $\alpha$, tumor necrosis factor- $\alpha$; COL1A1, collagen 1 type $\alpha 1 ; \alpha$-SMA, $\alpha$-smooth muscle actin; BMMSC/SC, bone marrow-derived mesenchymal stem cells; BA, biliary atresia.

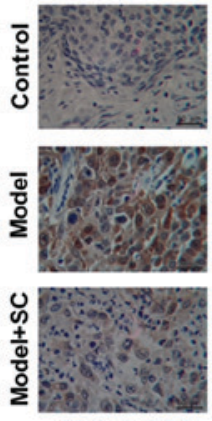

Collagen IV

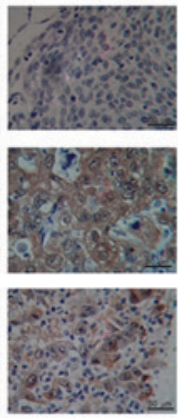

LN

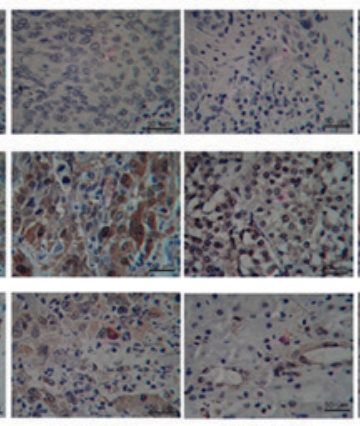

$\alpha$-SMA

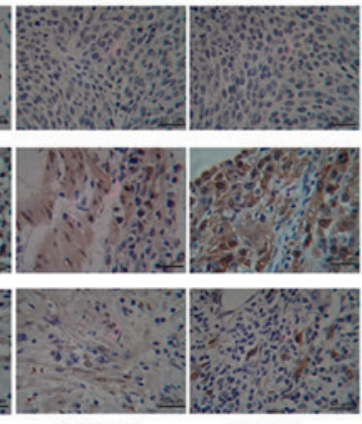

TGF- $\beta 1$

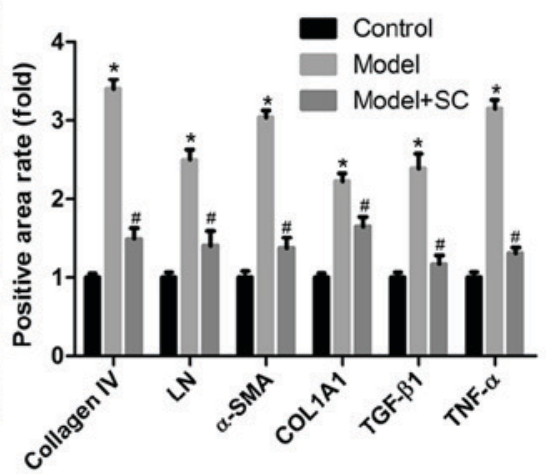

Figure 4. Immunohistochemistry analysis of the expression of proteins associated with liver fibrosis. Protein expression in liver tissues from the different groups: Control, BA, model and BA + BMMSC (model + SC). Magnification, $\mathrm{x} 100$; scale bars $=50 \mu \mathrm{m} .{ }^{*} \mathrm{P}<0.05$ vs. control group; ${ }^{*} \mathrm{P}<0.05$ vs. BA model group. LN, laminin; TGF- $\beta$, transforming growth factor- $\beta$; TNF- $\alpha$, tumor necrosis factor- $\alpha$; COL1A1, collagen 1 type $\alpha 1 ; \alpha$-SMA, $\alpha$-smooth muscle actin; BMMSC/SC, bone marrow-derived mesenchymal stem cells; BA, biliary atresia.
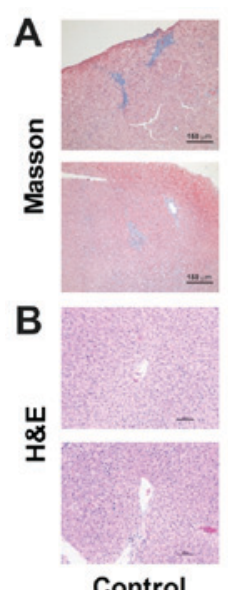
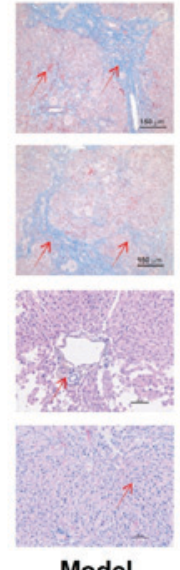
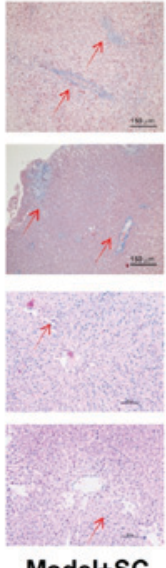

Model+SC

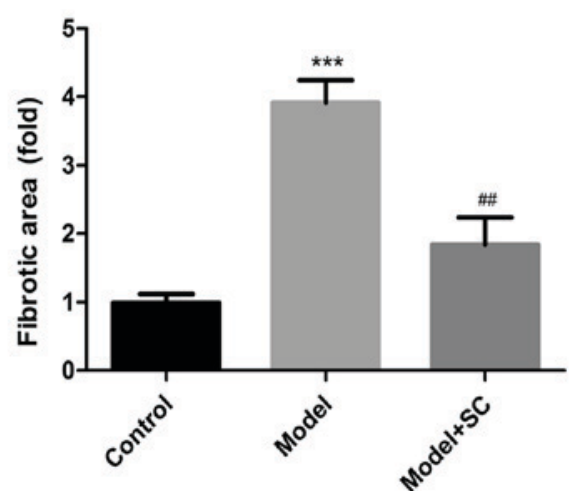

Figure 5. Histological analyses of livers from the control, BA model and BA + BMMSC (model + SC) treatment groups. (A) Masson's trichrome staining. (B) H\&E staining. Arrows indicate mononuclear cellular infiltration and marked fibrosis. Magnification, $\mathrm{x} 100$; scale bars $=150 \mu \mathrm{m}$. ${ }^{* * * *} \mathrm{P}<0.001 \mathrm{vs}$. control group; ${ }^{\# \#} \mathrm{P}<0.01$ vs. BA model group. H\&E, hematoxylin and eosin; BMMSC/SC, bone marrow-derived mesenchymal stem cells; BA, biliary atresia.

metabolism and inhibited oxidative stress and alleviated liver fibrosis of the RRV-induced murine model of BA. Our results were consistent with those of a previous study (16). Our study points to a potentially novel approach to ameliorate the fibrotic response of BA patients.
Besides, we showed that the administration of RRV led to a parallel increase of MDA, an oxidative stress marker, with a subsequent decrease of antioxidant markers (GSH and SOD). Active oxygen species play essential roles in host defense mechanisms against disease. However, excessive production of 
active oxygen species caused by chronic inflammation may lead to tissue injury and disease progression (17). In many BA patients, Kasai's hepatic portoenterostomy fails to resolve upregulation of oxidative stress, even in cases of mild liver dysfunction without jaundice (18). The aforementioned finding suggests that antioxidant therapy may be necessary for the treatment of BA patients. And our study demonstrated that BMMSCs transplantation significantly rescued the decrease of GSH and SOD and increase of MDA induced by RRV-administration, indicating the important value of BMMSCs in the treatment of BA. LN, an extracellular matrix component, is a major non-collagenous glycoprotein synthesized by hepatic stellate cells (HSCs) and deposited in the basement membrane of the liver (19). Previous study revealed that elevated levels of LN were correlated with the degree of perisinusoidal fibrosis (20). In the present study, the liver content of LN was significantly increased in the RRV-induced BA model, whereas it was significantly decreased after BMMSC treatment. In addition to LN, collagen, another extracellular matrix protein, plays an important role in fibrosis (21). Accordingly, we determined the effect of BMMSC therapy on the collagen content of liver tissue. Both collagen IV and COL1A1 were highly expressed in liver tissues of the model group, and the BMMSC treatment markedly reduced their expression. As demonstrated in previous research, activated HSCs are a major source of matrix proteins in diseased livers (22). Accordingly, we evaluated the formation of $\alpha$-SMA, an indicator of HSC activation (22), by immunohistochemical staining and western blotting of liver samples. The results showed that $\alpha$-SMA expression was elevated in the BA group, whereas it was clearly reduced in the BA BMMSC-treated group.

MSCs exert inhibitory effects on monocytes, dendritic cells, macrophages, and natural killer (NK) cells, which are part of the innate immune system. Besides, MSCs inhibit the maturation of monocytes into dendritic cells, which play a role in antigen presentation to naive T-cells and repress the secretion of TNF- $\alpha$, INF- $\gamma$, and interleukin-12 by dendritic cells and increase their secretion of interleukin-10, thereby reducing their pro-inflammatory potential (23). In contrast to MSCs, HSC activation in response to injury results in the secretion of TGF- $\beta 1$, which has potent fibrogenic effects in both autocrine and paracrine patterns (24). However, BMMSCs could suppress the activity of HSCs and reduce the secretion of specific cytokines, such as TGF- $\beta 1$ and interleukin-6 (24). In accordance with this finding, our study revealed that treatment with BMMSCs significantly inhibited inflammatory reactions in the liver by decreasing the secretion of pro-inflammatory factors, such as TNF- $\alpha$ and TGF- $\beta 1$.

Overall, the results of the present study point to the potential beneficial role of BMMSCs in the inhibition of fibrosis progression induced by RRV in mice.

\section{Acknowledgements}

Not applicable.

\section{Funding}

The present was supported by the National Natural Science Foundation of China (grant nos. 81460118, 81760115 and 81660092), Education Department Scientific Research
Foundation (grant no. GJJ14769) and Health Development Planning Commission Science Foundation of Jiangxi Province (grant no. 2012A135).

\section{Availability of data and materials}

The datasets used and/or analyzed during the current study are available from the corresponding author on reasonable request.

\section{Authors' contributions}

TX and SZ conceived and designed the study. JL, YC, JX, HH and ZL performed the experiments. $\mathrm{LY}$ and $\mathrm{JH}$ performed the histological examination of the liver. ZL and YX analyzed and interpreted the data. JL and YC wrote the paper. ZL, YX, LY and $\mathrm{JH}$ reviewed and edited the manuscript. All authors read and approved the manuscript.

\section{Ethics approval and consent to participate}

The present study was reviewed and approved by the Ethics Committee of the Jiangxi Provincial Children's Hospital.

\section{Patient consent for publication}

Not applicable.

\section{Competing interests}

The authors declare that they have no competing interest.

\section{References}

1. Hartley JL, Davenport M and Kelly DA: Biliary atresia. Lancet 374: 1704-1713, 2009.

2. Mieli-Vergani G and Vergani D: Biliary atresia. Semin Immunopathol 31: 371-381, 2009.

3. Sira MM, Taha M and Sira AM: Common misdiagnoses of biliary atresia. Eur J Gastroenterol Hepatol 26: 1300-1305, 2014.

4. You Z, Wen J, Cheng L, Ye H and Li B: Screening of targeted genes in extrahepatic bile ducts of mice with experimental biliary atresia. Mol Med Rep 12: 4326-4331, 2015.

5. Zagory JA, Nguyen MV and Wang KS: Recent advances in the pathogenesis and management of biliary atresia. Curr Opin Pediatr 27: 389-394, 2015.

6. Li Y, Wu Q, Wang Y, Li L, Bu H and Bao J: Senescence of mesenchymal stem cells (Review). Int J Mol Med 39: 775-782, 2017.

7. Wang H, Zhang H, Huang B, Miao G, Yan X, Gao G, Luo Y, Chen H, Chen W and Yang L: Mesenchymal stem cells reverse highfat dietinduced nonalcoholic fatty liver disease through suppression of CD4+ T lymphocytes in mice. Mol Med Rep 17: 3769-3774, 2018.

8. Sioud M, Mobergslien A, Boudabous A and Floisand Y: Mesenchymal stem cell-mediated T cell suppression occurs through secreted galectins. Int J Oncol 38: 385-390, 2011.

9. Berardis S, Lombard C, Evraerts J, El Taghdouini A, Rosseels V, Sancho-Bru P, Lozano JJ, van Grunsven L, Sokal E and Najimi M: Gene expression profiling and secretome analysis differentiate adult-derived human liver stem/progenitor cells and human hepatic stellate cells. PLoS One 9: e86137, 2014.

10. Zhang R, Lin Z, Lui VCH, Wong KKY, Tam PKH, Lee P, Lok CN, Lamb JR, Chen Y and Xia H: Silver nanoparticle treatment ameliorates biliary atresia syndrome in rhesus rotavirus inoculated mice. Nanomedicine 13: 1041-1050, 2017.

11. Rojas M, Xu J, Woods CR, Mora AL, Spears W, Roman J and Brigham KL: Bone marrow-derived mesenchymal stem cells in repair of the injured lung. Am J Respir Cell Mol Biol 33: 145-152, 2005. 
12. Mohanty SK, Donnelly B, Dupree P, Lobeck I, Mowery S, Meller J, McNeal M and Tiao G: A point mutation in the rhesus rotavirus VP4 protein generated through a rotavirus reverse genetics system attenuates biliary atresia in the murine model. J Virol 91: e00510, 2017.

13. Bezerra JA: Potential etiologies of biliary atresia. Pediatr Transplantat 9: 646-651, 2005.

14. Petersen C, Grasshoff S and Luciano L: Diverse morphology of biliary atresia in an animal model. J Hepatol 28: 603-607, 1998.

15. Riepenhoff-Talty M, Schaekel K, Clark HF, Mueller W, Uhnoo I, Rossi T, Fisher J and Ogra PL: Group A rotaviruses produce extrahepatic biliary obstruction in orally inoculated newborn mice. Pediatr Research 33: 394-399, 1993.

16. Elmahdy NA, Sokar SS, Salem ML, Sarhan NI and Abou-Elela SH: Anti-fibrotic potential of human umbilical cord mononuclear cells and mouse bone marrow cells in CCl4-induced liver fibrosis in mice. Biomed Pharmacother 89: 1378-1386, 2017.

17. Cadenas E: Biochemistry of oxygen toxicity. Annu Rey Biochem 58: 79-110, 1989.

18. Asakawa T, Tanaka Y, Asagiri K, Kobayashi H, Tanikawa K and Yagi M: Oxidative stress profile in the post-operative patients with biliary atresia. Pediatr Surg Int 25: 93-97, 2009.

19. Tang N, Zhang Y, Liu Z, Fu T, Liang Q and Ai X: Correlation analysis between four serum biomarkers of liver fibrosis and liver function in infants with cholestasis. Biomed Rep 5: 107-112, 2016.
20. Baranova A, Lal P, Birerdinc A and Younossi ZM: Non-invasive markers for hepatic fibrosis. BMC Gastroenterol 11: 91, 2011.

21. Wang B, Komers R, Carew R, Winbanks CE, Xu B Herman-Edelstein M, Koh P, Thomas M, Jandeleit-Dahm K, Gregorevic P, et al: Suppression of microRNA-29 expression by TGF- $\beta 1$ promotes collagen expression and renal fibrosis. J Am Soc Nephrol 23: 252-265, 2012

22. Williams EJ, Benyon RC, Trim N, Hadwin R, Grove BH, Arthur MJ, Unemori EN and Iredale JP: Relaxin inhibits effective collagen deposition by cultured hepatic stellate cells and decreases rat liver fibrosis in vivo. Gut 49: 577-583, 2001.

23. Jiang XX, Zhang Y, Liu B, Zhang SX, Wu Y, Yu XD and Mao N: Human mesenchymal stem cells inhibit differentiation and function of monocyte-derived dendritic cells. Blood 105: 4120-4126, 2005.

24. Jang YO, Jun BG, Baik SK, Kim MY and Kwon SO: Inhibition of hepatic stellate cells by bone marrow-derived mesenchymal stem cells in hepatic fibrosis. Clin Mol Hepatol 21: 141-149, 2015. 